\title{
Convolutions and applications for the offset linear canonical transform via Hermite weights
}

Cite as: AIP Conference Proceedings 2046, 020014 (2018); https://doi.org/10.1063/1.5081534 Published Online: 04 December 2018

L. P. Castro, L. T. Minh, and N. M. Tuan

\section{ARTICLES YOU MAY BE INTERESTED IN}

New convolutions for an oscillatory integral operator on the half-line AIP Conference Proceedings 2046, 020015 (2018); https://doi.org/10.1063/1.5081535

On integral operators and equations generated by cosine and sine Fourier transforms AIP Conference Proceedings 2046, 020013 (2018); https://doi.org/10.1063/1.5081533

Stabilities for a class of higher order integro-differential equations AIP Conference Proceedings 2046, 020012 (2018); https://doi.org/10.1063/1.5081532
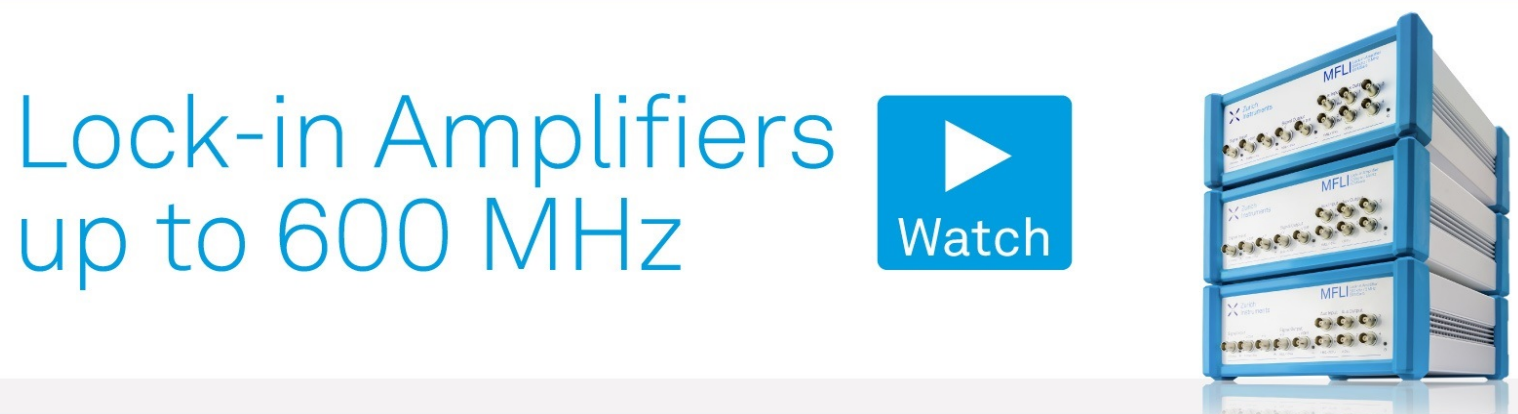


\title{
Convolutions and Applications for the Offset Linear Canonical Transform Via Hermite Weights
}

\author{
L.P. Castro ${ }^{1, a), b)}$, L.T. Minh ${ }^{2, c)}$ and N.M. Tuan ${ }^{3, d)}$ \\ ${ }^{1}$ Center for Research and Development in Mathematics and Applications (CIDMA), Department of Mathematics, \\ University of Aveiro, Aveiro, Portugal. \\ ${ }^{2}$ Department of Mathematics, Ha Noi Architectural University, Km 10, Nguyen Trai Rd., Thanh Xuan Dist., Ha Noi, \\ Vietnam. \\ ${ }^{3}$ Department of Mathematics, College of Education, Viet Nam National University, G7 Build., 144 Xuan Thuy Rd., \\ Cau Giay Dist., Hanoi, Vietnam.

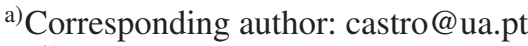 \\ b)URL: http://sweet.ua.pt/castro/ \\ c) tienminh.hau@gmail.com \\ d)nguyentuan@vnu.edu.vn
}

\begin{abstract}
The main purpose of this paper is to present three new convolutions for the offset linear canonical transform, with the Hermite weights, and to illustrated their potential applications. In view of this, new factorization theorems are obtained and new Young's convolution inequalities will be introduced. Within the more applied side, the way to design filters (including multiplicative filters in the time domain) is also discussed in the last section.
\end{abstract}

\section{INTRODUCTION}

The offset linear canonical transform (OLCT) (see [7]) of a signal $f(t)$ with real parameters $A=\left(a, b, c, d, u_{0}, \omega_{0}\right)$, (satisfying $a d-b c=1$ ) is defined as

$$
F_{A}(u):=\mathbb{O}_{A}\{f(t)\}(u):=\left\{\begin{array}{l}
\int_{\mathbb{R}} f(t) \mathcal{K}_{A}(u, t) d t, \quad b \neq 0 \\
\sqrt{d} e^{j \frac{c d}{2}\left(u-u_{0}\right)^{2}+j \omega_{0} u} f\left(d\left(u-u_{0}\right)\right), \quad b=0,
\end{array}\right.
$$

where $\mathcal{K}_{A}(u, t):=K_{A} e^{j\left(\frac{d}{2 b} u^{2}-\frac{1}{b} t u+\frac{a}{2 b} t^{2}+\frac{\left(b \omega_{0}-d u_{0}\right)}{b} u+\frac{u_{0}}{b} t\right)}$, and $K_{A}=\frac{e^{\frac{j d u_{0}^{2}}{2 b}}}{\sqrt{2 \pi b j}}$. The inverse of the OLCT is given by

$$
f(t)=\mathbb{O}_{A^{-1}}\left\{F_{A}(u)\right\}(t)=C \int_{\mathbb{R}} F_{A}(u) \mathcal{K}_{A^{-1}}(u, t) d u,
$$

where $A^{-1}=\left(d,-b,-c, a, b \omega_{0}-d u_{0}, c u_{0}-a \omega_{0}\right)$, and $C=e^{j \frac{1}{2}\left(c d u_{0}^{2}-2 a d u_{0} \omega_{0}+a b \omega_{0}^{2}\right)}$. In this paper, we will always consider $b \neq 0$ since the OLCT becomes a chirp multiplication operation otherwise. We recall that the Fourier transform and its inverse are defined by $\Psi_{F T}(f(t))(u)=\int_{\mathbb{R}} f(t) e^{-j u t} d t$ and $f(t)=\frac{1}{2 \pi} \int_{\mathbb{R}} \Psi_{F T}(f(t))(u) \cdot e^{j u t} d u$, respectively. If $f, h \in L^{1}(\mathbb{R})$, then the classic (Fourier) convolution in the time domain is expressed as

$$
(f * h)(t):=\int_{\mathbb{R}} f(\tau) h(t-\tau) d \tau
$$

and the factorization property as follows

$$
\Psi_{F T}\{(f * h)(t)\}(u)=\Psi_{F T}\{f(t)\}(u) \cdot \Psi_{F T}\{h(t)\}(u) .
$$


For any real number $\lambda \neq 0$, we have

$$
(f * h)(\lambda t)=\lambda f(\lambda t) * h(\lambda t) .
$$

We also have the Young's inequality (see [2]). If $f \in L^{p}(\mathbb{R}), h \in L^{q}(\mathbb{R})$, and $\frac{1}{p}+\frac{1}{q}=\frac{1}{r}+1$ (with $p, q, r \geqslant 1$ ). Then, the following inequality holds

$$
\|f * h\|_{r} \leqslant C_{1} \cdot\|f\|_{p} \cdot\|h\|_{q}, \text { for some } C_{1}>0 .
$$

We notice that when $u_{0}=\omega_{0}=0, \mathbb{O}_{A}$ is the well-known linear canonical transform (LCT) (see [4]). Remind that if $A=(a, b, c, d, 0,0),|a+d|<2$, and $\phi_{n}(t), \mu_{n}$ are the eigenfunctions and the eigenvalues of the OLCT (or the LCT) (see [6]), then we have

$$
\mu_{n} \phi_{n}(u)=\mathbb{O}_{A}\left\{\phi_{n}(t)\right\}(u)
$$

where

$$
\phi_{n}(t):=\frac{1}{\sqrt{\beta 2^{n} n ! \sqrt{\pi}}} e^{-\frac{(1+j \alpha)}{2 \beta^{2}} t^{2}} H_{n}\left(\frac{t}{\beta}\right), \quad \mu_{n}:=e^{-j\left(\frac{1}{2}+n\right) \theta} \quad(n \in \mathbb{N})
$$

and $H_{n}$ is the $n$-th Hermite polynomial. The constants $\alpha, \beta, \theta$ can be taken from

$$
\alpha:=\frac{\operatorname{sgn}(b) \cdot(a-d)}{\sqrt{4-(a+d)^{2}}}, \beta:=\frac{2|b|}{\sqrt{4-(a+d)^{2}}}, \theta:=\cos ^{-1}\left(\frac{a+d}{2}\right) .
$$

Throughout this paper, for convenience, we denote

$$
\mathcal{E}_{A}(t):=e^{j\left(\frac{a}{2 b} t^{2}+\frac{u_{0}}{b} t\right)}, \quad \bar{f}(t):=\mathcal{E}_{A}(t) f(t), \quad \mathcal{E}_{A}^{m}(t):=e^{j m t} \mathcal{E}_{A}(t) \quad(m \in \mathbb{R}) .
$$

The identity (1) becomes

$$
F_{A}(u)=\mathbb{O}_{A}\{f(t)\}(u)=K_{A} e^{j\left(\frac{d}{2 b} u^{2}+\frac{\left(b \omega_{0}-d u_{0}\right)}{b} u\right)} \int_{\mathbb{R}} \bar{f}(t) e^{-\frac{j u t}{b}} d t .
$$

This paper is divided into four sections and organized as follows. In Section 2, we introduce the relationship between the Hermite functions and the OLCT, which are displayed in Theorem 1 and Theorem 2. Three new convolutions for the OLCT with the Hermite weights and their product theorems are studied in the Section 3. Some special cases of these convolutions are also obtained. In the last section, we propose some applications of these convolutions as well as new Young's convolution inequalities and designing multiplicative filter in the time domain.

\section{HERMITE FUNCTIONS AND THE OLCT}

For $\lambda \neq 0$, it is easy to realize that $1=a d-b c=(a \lambda)\left(\frac{d}{\lambda}\right)-\left(\frac{b}{\lambda}\right)(c \lambda)$. Let $\lambda \neq 0$, and the parameters $A_{\lambda}:=$ $\left(a \lambda, \frac{b}{\lambda}, c \lambda, \frac{d}{\lambda}, 0,0\right)$ satisfy

$$
\left|a \lambda+\frac{d}{\lambda}\right|<2
$$

Under the condition (10), let $\phi_{n}^{\lambda}(t), \mu_{n}^{\lambda}$ be the eigenfunctions and the corresponding eigenvalues of the OLCT with parameters $A_{\lambda}=\left(a \lambda, \frac{b}{\lambda}, c \lambda, \frac{d}{\lambda}, 0,0\right)$. We then have $\mu_{n}^{\lambda} \cdot \phi_{n}^{\lambda}(u)=\mathbb{O}_{A_{\lambda}}\left\{\phi_{n}^{\lambda}(t)\right\}(u)$. The eigenfunctions $\phi_{n}^{\lambda}(t)$ and the eigenvalues $\mu_{n}^{\lambda}$ corresponding parameters $A_{\lambda}$ can be calculated as in (8).

Theorem 1 Let the parameters $A_{1}=(a, b, c, d, 0,0)$, and one of the following conditions is satisfied:

(i) $|a+d|<2$; (ii) $|a+d| \geqslant 2$ and $1-a d>0$.

Then, there exists a constant $\lambda>0$ such that the following relation holds

$$
\phi_{n}^{\lambda}(u)=\frac{1}{\mu_{n}^{\lambda} \cdot \sqrt{\lambda}} \mathbb{O}_{A_{1}}\left\{\phi_{n}^{\lambda}\left(\frac{t}{\lambda}\right)\right\}(u) \text {. }
$$


Proof. If $|a+d|<2$ then from relation (7) we choose $\lambda=1$. Thus, (11) is fulfilled.

If $|a+d| \geqslant 2$ and $1-a d>0$. By changing the variable $t=\lambda \tau(\lambda>0)$, we get

$$
\mathcal{K}_{A_{1}}(u, t)=K_{A_{1}} e^{j\left(\frac{d}{2 b} u^{2}-\frac{1}{b} t u+\frac{a}{2 b} t^{2}\right)}=K_{A_{1}} e^{j\left(\frac{d}{2 b} u^{2}-\frac{\lambda}{b} \tau u+\frac{a}{2 b} \lambda^{2} \tau^{2}\right)}=K_{A_{1}} e^{j\left(\frac{\left(\frac{d}{\lambda}\right)}{2\left(\frac{b}{\lambda}\right)} u^{2}-\frac{1}{\left(\frac{b}{\lambda}\right)} \tau u+\frac{a \lambda}{2\left(\frac{b}{\lambda}\right)} \tau^{2}\right)}=\frac{1}{\sqrt{\lambda}} \mathcal{K}_{A_{\lambda}}(u, \tau) .
$$

It follows

$$
\mathcal{K}_{A_{\lambda}}(u, \tau)=\sqrt{\lambda} \mathcal{K}_{A_{1}}(u, t) .
$$

Assume that the condition (10) is satisfied. We then have

$$
\left(a \lambda^{2}-2 \lambda+d\right)\left(a \lambda^{2}+2 \lambda+d\right)<0 .
$$

If $a=0$, then from (13) we derive $|\lambda|>\frac{|d|}{2}$. Thus, there exists $\lambda>0$ such as (10) is satisfied. If $a \neq 0$, since $\delta=1-a d>$ 0 then we denote $\lambda_{1}<\lambda_{2}<\lambda_{3}<\lambda_{4}$ be four solutions of the following equation $\left(a \lambda^{2}-2 \lambda+d\right)\left(a \lambda^{2}+2 \lambda+d\right)=0$. Solving this equation, we receive

$$
\lambda_{i} \in\left\{\frac{-1 \pm \sqrt{\delta}}{a}, \frac{1 \pm \sqrt{\delta}}{a}\right\}, i \in\{1,2,3,4\}
$$

From (13), we deduce $\lambda \in\left(\lambda_{1}, \lambda_{2}\right) \cup\left(\lambda_{3}, \lambda_{4}\right)$, and $\lambda_{4}=\frac{1+\sqrt{\delta}}{|a|}>0$. Hence, there exists $\lambda>0$ such that (10) is fulfilled. Therefore, the OLCT with parameters $A_{\lambda}$ has the eigenfunctions $\phi_{n}^{\lambda}(t)$ and the eigenvalues $\mu_{n}^{\lambda}$ :

$$
\mu_{n}^{\lambda} \cdot \phi_{n}^{\lambda}(u)=\int_{\mathbb{R}} \mathcal{K}_{A_{\lambda}}(u, \tau) \cdot \phi_{n}^{\lambda}(\tau) d \tau
$$

Substituting the relation (12) into (14) results in

$$
\mu_{n}^{\lambda} \cdot \phi_{n}^{\lambda}(u)=\sqrt{\lambda} \int_{\mathbb{R}} \mathcal{K}_{A_{1}}(u, t) \cdot \phi_{n}^{\lambda}\left(\frac{t}{\lambda}\right) d\left(\frac{t}{\lambda}\right) .
$$

That implies $\phi_{n}^{\lambda}(u)=\frac{1}{\mu_{n}^{\lambda} \cdot \sqrt{\lambda}} \int_{\mathbb{R}} \mathcal{K}_{A_{1}}(u, t) \cdot \phi_{n}^{\lambda}\left(\frac{t}{\lambda}\right) d t$. Hence $\phi_{n}^{\lambda}(u)=\frac{1}{\mu_{n}^{\lambda} \cdot \sqrt{\lambda}} \mathbb{O}_{A_{1}}\left\{\phi_{n}^{\lambda}\left(\frac{t}{\lambda}\right)\right\}(u)$. The proof is completed.

Theorem 2 Let $A=\left(a, b, c, d, u_{0}, \omega_{0}\right)$, and one of the following conditions be fulfilled: $(i)|a+d|\langle 2 ;(i i)|a+d| \geqslant$ $2, a+d \neq 2$ and $1-a d>0$. Then, there exists a positive constant $\lambda$, such that the following relation holds

$$
e^{j m_{1} u} \cdot \phi_{n}^{\lambda}\left(u-m_{2}\right)=\frac{e^{j\left(m_{3}-\frac{d u_{0}^{2}}{2 b}\right)}}{\mu_{n}^{\lambda} \cdot \sqrt{\lambda}} \mathbb{O}_{A}\left\{e^{j m_{1} t} \cdot \phi_{n}^{\lambda}\left(\frac{t-m_{2}}{\lambda}\right)\right\}(u),
$$

provided

$$
\left\{\begin{array}{l}
m_{1}=\frac{(a-1)\left(b \omega_{0}-d u_{0}\right)+u_{0}(1-d)}{b(a+d-2)} \\
m_{2}=-\frac{b \omega_{0}-d u_{0}+u_{0}}{a+d-2} \\
m_{3}=\frac{\left(b \omega_{0}-d u_{0}+u_{0}\right)^{2}}{2 b(a+d-2)}
\end{array}\right.
$$

Proof. We realize that

$$
\begin{aligned}
\int_{\mathbb{R}} \mathcal{K}_{A}(u, t) \cdot e^{j\left(m_{1} t+m_{3}\right)} e^{-j m_{1} u} \cdot \phi_{n}^{\lambda}\left(\frac{t-m_{2}}{\lambda}\right) d t & =K_{A} \int_{\mathbb{R}} e^{j\left(\frac{d}{2 b} u^{2}-\frac{1}{b} t u+\frac{a}{2 b} t^{2}+\frac{\left(b \omega_{0}-d u_{0}\right)}{b} u+\frac{u_{0}}{b} t\right)} e^{j\left(m_{1} t+m_{3}\right)} e^{-j m_{1} u} \cdot \phi_{n}^{\lambda\left(\frac{t-m_{2}}{\lambda}\right) d t} \\
& =K_{A} \int_{\mathbb{R}} e^{j\left(\frac{d}{2 b} u^{2}-\frac{1}{b} t u+\frac{a}{2 b} t^{2}+\left(\frac{b \omega_{0}-d u_{0}}{b}-m_{1}\right) u+\left(\frac{u_{0}}{b}+m_{1}\right) t+m_{3}\right)} \cdot \phi_{n}^{\lambda\left(\frac{t-m_{2}}{\lambda}\right) d t .}
\end{aligned}
$$

Let

$$
\frac{d}{2 b} u^{2}-\frac{1}{b} t u+\frac{a}{2 b} t^{2}+\left(\frac{b \omega_{0}-d u_{0}}{b}-m_{1}\right) u+\left(\frac{u_{0}}{b}+m_{1}\right) t+m_{3}=\frac{d}{2 b}\left(u-m_{2}\right)^{2}-\frac{1}{b}\left(t-m_{2}\right)\left(u-m_{2}\right)+\frac{a}{2 b}\left(t-m_{2}\right)^{2},
$$


then

$$
\left\{\begin{array}{l}
m_{1}-\frac{d-1}{b} m_{2}=\frac{b \omega_{0}-d u_{0}}{b} \\
m_{1}+\frac{a-1}{b} m_{2}=-\frac{u_{0}}{b} \\
m_{3}=m_{2}^{2}\left(\frac{d}{2 b}-\frac{1}{b}+\frac{a}{2 b}\right)
\end{array}\right.
$$

Remind that $a+d \neq 2$. Then, the solution of this system equations is given as

$$
\left\{\begin{array}{l}
m_{1}=\frac{(a-1)\left(b \omega_{0}-d u_{0}\right)+u_{0}(1-d)}{b(a+d-2)} \\
m_{2}=-\frac{b \omega_{0}-d u_{0}+u_{0}}{a+d-2} \\
m_{3}=\frac{\left(b \omega_{0}-d u_{0}+u_{0}\right)^{2}}{2 b(a+d-2)}
\end{array}\right.
$$

Substituting equation (18) into equation (17), we obtain

$$
\int_{\mathbb{R}} \mathcal{K}_{A}(u, t) e^{j\left(m_{1} t+m_{3}\right)} e^{-j m_{1} u} \phi_{n}^{\lambda}\left(\frac{t-m_{2}}{\lambda}\right) d t=e^{\frac{j d u_{0}^{2}}{2 b}} \int_{\mathbb{R}} \mathcal{K}_{A_{1}}\left(u-m_{2}, t-m_{2}\right) \phi_{n}^{\lambda}\left(\frac{t-m_{2}}{\lambda}\right) d t=e^{\frac{j d u_{0}^{2}}{2 b}} \mathbb{O}_{A_{1}}\left\{\phi_{n}^{\lambda}\left(\frac{t}{\lambda}\right)\right\}\left(u-m_{2}\right) .
$$

Thanks to equation (11), we derive $\int_{\mathbb{R}} \mathcal{K}_{A}(u, t) \cdot e^{j\left(m_{1} t+m_{3}\right)} e^{-j m_{1} u} \cdot \phi_{n}^{\lambda}\left(\frac{t-m_{2}}{\lambda}\right) d t=e^{\frac{j d u_{0}^{2}}{2 b}} \cdot \mu_{n}^{\lambda} \cdot \sqrt{\lambda} \cdot \phi_{n}^{\lambda}\left(u-m_{2}\right)$, which implies that $e^{-j m_{3}} e^{\frac{j d u_{0}^{2}}{2 b}} \mu_{n}^{\lambda} \cdot \sqrt{\lambda} \cdot \phi_{n}^{\lambda}\left(u-m_{2}\right)=\int_{\mathbb{R}} \mathcal{K}_{A}(u, t) \cdot e^{j m_{1} t} e^{-j m_{1} u} \cdot \phi_{n}^{\lambda}\left(\frac{t-m_{2}}{\lambda}\right) d t$. This means

$$
e^{j m_{1} u} \cdot \phi_{n}^{\lambda}\left(u-m_{2}\right)=\frac{e^{j\left(m_{3}-\frac{d u_{0}^{2}}{2 b}\right)}}{\mu_{n}^{\lambda} \cdot \sqrt{\lambda}} \mathbb{O}_{A}\left\{e^{j m_{1} t} \cdot \phi_{n}^{\lambda}\left(\frac{t-m_{2}}{\lambda}\right)\right\}(u) .
$$

The theorem is achieved.

Remark 1 If $a+d=2$, and $b \omega_{0}-(1-a) u_{0}=0$, then the relation (15) holds for the following conditions $m_{1}=\frac{(1-a)}{b} m_{2}-\frac{u_{0}}{b}, m_{2} \in \mathbb{R}$ and $m_{3}=0$.

Example 1 Consider the case $A=\left(-\frac{2}{3}, \frac{1}{3},-9,3,1,3\right), A_{1}=\left(-\frac{2}{3}, \frac{1}{3},-9,3,0,0\right)$. Since $|a+d|=\frac{7}{3}>2$, then it is easily seen that

$$
\lambda \in\left(\frac{-3-3 \sqrt{3}}{2}, \frac{3-3 \sqrt{3}}{2}\right) \cup\left(\frac{3 \sqrt{3}-3}{2}, \frac{3+3 \sqrt{3}}{2}\right) .
$$

If we choose $\lambda=\frac{3}{2}$, then $A_{\lambda}=\left(-1, \frac{2}{9},-\frac{27}{2}, 2,0,0\right)$. The Hermite functions and the values $\mu_{n}^{\lambda}$ can be expressed as

$$
\phi_{n}^{\lambda}(t)=\frac{3}{2 \sqrt{2^{n} n ! \sqrt{3 \pi}}} e^{-\frac{243(1-j \sqrt{3})}{32} t^{2}} H_{n}\left(\frac{9 \sqrt{3} t}{4}\right), \quad \mu_{n}^{\lambda}=e^{-j\left(\frac{1}{2}+n\right) \frac{\pi}{3}} \quad(n \in \mathbb{N}) .
$$

Therefore, the relation (11) becomes $\phi_{n}^{\lambda}(t)=\sqrt{\frac{2}{3}} e^{j\left(\frac{1}{2}+n\right) \frac{\pi}{3}} \cdot \mathbb{O}_{A_{1}}\left\{\phi_{n}^{\lambda}\left(\frac{2 t}{3}\right)\right\}(u)$. From (16), we obtain $m_{1}=12, m_{2}=3, m_{3}=$ $\frac{9}{2}$. The relation (15) gives $e^{12 j t} \phi_{n}^{\lambda}(t-3)=\sqrt{\frac{2}{3}} e^{j\left(\frac{1}{2}+n\right) \frac{\pi}{3}} \cdot \mathbb{O}_{A}\left\{e^{12 j t} \phi_{n}^{\lambda}\left(\frac{2 t-6}{3}\right)\right\}(u)$.

\section{CONVOLUTIONS FOR THE OFFSET LINEAR CANONICAL TRANSFORM WITH HERMITE WEIGHTS}

In this section, the space $L^{p}(\mathbb{R})$ will be endowed with the norm $\|\cdot\|_{p}$ defined by $\|f\|_{p}:=\left(\int_{\mathbb{R}}|f(t)|^{p} d t\right)^{\frac{1}{p}}, p \geqslant 1$. Assume that the conditions of Theorem 1 and Theorem 2 are satisfied. The convolution for the OLCT of two signals $f(t)$ and $h(t)$ is defined by

$$
(f \stackrel{1}{\otimes} h)(t):=\frac{K_{A}^{2} e^{j\left(m_{3}-\frac{d u_{0}^{2}}{2 b}\right)}\left(\mathcal{E}_{A}(t)\right)^{-1}}{\mu_{n}^{\lambda} \cdot \sqrt{\lambda}} \int_{\mathbb{R}^{2}} \bar{f}(\tau) \bar{h}(v) \cdot \mathcal{E}_{A}^{m_{1}}(t-\tau-v) \phi_{n}^{\lambda}\left(\frac{t-\tau-v-m_{2}}{\lambda}\right) d \tau d v,
$$

provided that the integral in (19) is well-defined. Moreover, if $f, h \in L^{1}(\mathbb{R})$, then the function defined in (19) belongs to $L^{1}(\mathbb{R})$, and $\|f \stackrel{1}{\otimes} h\|_{1} \leqslant C_{2}\|f\|_{1} \cdot\|h\|_{1}$, where $C_{2}$ is a positive constant. We have the following product theorem. 
Theorem 3 Assume that $f, h \in L^{1}(\mathbb{R}), F_{A}$ and $H_{A}$ denote the OLCT of the signals $f(t)$ and $h(t)$ with parameters A, respectively. We have

$$
\mathbb{O}_{A}\{(f \stackrel{1}{\otimes} h)(t)\}(t)=e^{j m_{1} u} e^{-j\left(\frac{d}{b} u^{2}+2 \frac{\left(b \omega_{0}-d u_{0}\right)}{b} u\right)} \cdot \phi_{n}^{\lambda}\left(u-m_{2}\right) \cdot F_{A}(u) \cdot H_{A}(u) .
$$

Moreover, if $e^{j m_{1} u} e^{-j\left(\frac{d}{b} u^{2}+2 \frac{\left(b \omega_{0}-d u_{0}\right)}{b} u\right)} \cdot \phi_{n}^{\lambda}\left(u-m_{2}\right) \cdot F_{A}(u) \cdot H_{A}(u) \in \mathbb{O}_{A}\left(L^{1}(\mathbb{R})\right)$, then

$$
(f \stackrel{1}{\otimes} h)(t)=\mathbb{O}_{A^{-1}}\left\{e^{j m_{1} u} e^{-j\left(\frac{d}{b} u^{2}+2 \frac{\left(b \omega_{0}-d u_{0}\right)}{b} u\right)} \cdot \phi_{n}^{\lambda}\left(u-m_{2}\right) \cdot F_{A}(u) \cdot H_{A}(u)\right\}(t) .
$$

Proof. Using the identity (9) and Theorem 2, we realize that

$$
\begin{aligned}
& e^{j m_{1} u} e^{-j\left(\frac{d}{b} u^{2}+2 \frac{\left(b \omega_{0}-d u_{0}\right)}{b} u\right)} \cdot \phi_{n}^{\lambda}\left(u-m_{2}\right) \cdot F_{A}(u) \cdot H_{A}(u) \\
& =e^{j m_{1} u} e^{-j\left(\frac{d}{b} u^{2}+2 \frac{\left(b \omega_{0}-d u_{0}\right)}{b} u\right)} \cdot \phi_{n}^{\lambda}\left(u-m_{2}\right) K_{A}^{2} e^{j\left(\frac{d}{b} u^{2}+\frac{2\left(b \omega_{0}-d u_{0}\right)}{b} u\right)} \int_{\mathbb{R}^{2}} \bar{f}(\tau) \bar{h}(v) e^{-\frac{j u \tau}{b}} e^{-\frac{j u v}{b}} d \tau d v \\
& =\frac{e^{j\left(m_{3}-\frac{d u_{0}^{2}}{2 b}\right)}}{\mu_{n}^{\lambda} \cdot \sqrt{\lambda}} K_{A}^{3} e^{j\left(\frac{d}{2 b} u^{2}+\frac{\left(b \omega_{0}-d u_{0}\right)}{b} u\right)} \int_{\mathbb{R}} e^{-\frac{j u t}{b}} \cdot \mathcal{E}_{A}^{m_{1}}(t) \cdot \phi_{n}^{\lambda}\left(\frac{t-m_{2}}{\lambda}\right) d t \int_{\mathbb{R}^{2}} \bar{f}(\tau) \bar{h}(v) e^{-\frac{j u \tau}{b}} e^{-\frac{j u v}{b}} d \tau d v \\
& =\frac{e^{j\left(m_{3}-\frac{d u_{0}^{2}}{2 b}\right)}}{\mu_{n}^{\lambda} \cdot \sqrt{\lambda}} K_{A}^{3} e^{j\left(\frac{d}{2 b} u^{2}+\frac{\left(b \omega_{0}-d u_{0}\right)}{b} u\right)} \int_{\mathbb{R}^{3}} e^{-j \frac{(t+\tau+v) u}{b}} \cdot \bar{f}(\tau) \bar{h}(v) \cdot \mathcal{E}_{A}^{m_{1}}(t) \cdot \phi_{n}^{\lambda}\left(\frac{t-m_{2}}{\lambda}\right) d \tau d v d t .
\end{aligned}
$$

By making $\tau=\tau, v=v$ and $s=t+\tau+v$, we obtain

$$
\begin{gathered}
e^{j m_{1} u} e^{-j\left(\frac{d}{b} u^{2}+2 \frac{\left(b \omega_{0}-d u_{0}\right)}{b} u\right)} \cdot \phi_{n}^{\lambda}\left(u-m_{2}\right) \cdot F_{A}(u) \cdot H_{A}(u) \\
=K_{A} e^{-j\left(\frac{d}{b} u^{2}+2 \frac{\left(b \omega_{0}-d u_{0}\right)}{b} u\right)} \int_{\mathbb{R}} e^{-\frac{j u s}{b}} \mathcal{E}_{A}(s)\left\{\frac{K_{A}^{2} e^{j\left(m_{3}-\frac{d u_{0}^{2}}{2 b}\right)}\left(\mathcal{E}_{A}(s)\right)^{-1}}{\mu_{n}^{\lambda} \cdot \sqrt{\lambda}} \int_{\mathbb{R}^{2}} \bar{f}(\tau) \bar{h}(v) \mathcal{E}_{A}^{m_{1}}(s-\tau-v) \cdot \phi_{n}^{\lambda}\left(\frac{s-\tau-v-m_{2}}{\lambda}\right) d \tau d v\right\} d s \\
=\int_{\mathbb{R}} \mathcal{K}_{A}(u, s)\left\{\frac{K_{A}^{2} e^{\left(m_{3}-\frac{d u_{0}^{2}}{2 b}\right)}\left(\mathcal{E}_{A}(s)\right)^{-1}}{\mu_{n}^{\lambda} \cdot \sqrt{\lambda}} \int_{\mathbb{R}^{2}} \bar{f}(\tau) \bar{h}(v) \cdot \mathcal{E}_{A}^{m_{1}}(s-\tau-v) \phi_{n}^{\lambda}\left(\frac{s-\tau-v-m_{2}}{\lambda}\right) d \tau d v\right\} d s \\
=\int_{\mathbb{R}} \mathcal{K}_{A}(u, s) \cdot(f \stackrel{1}{\otimes} h)(s) d s=\mathbb{O}_{A}\{(f \otimes h)\}(u) .
\end{gathered}
$$

The proof is concluded.

By using the same method as in Theorem 3, we derive the next result.

Theorem 4 Let $f, h \in L^{1}(\mathbb{R}), F_{A}$ and $H_{A}$ denote the OLCT of the signals $f(t)$ and $h(t)$ with parameters A, respectively. The transform

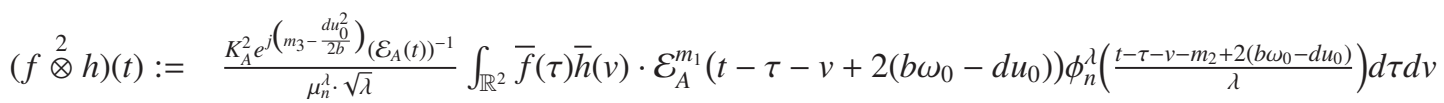

defines a convolution belonging $L^{1}(\mathbb{R})$, and turns possible the following factorization identity

$$
\mathbb{O}_{A}\{(f \stackrel{2}{\otimes} h)(t)\}(t)=e^{j m_{1} u} e^{-j \frac{d}{b} u^{2}} \cdot \phi_{n}^{\lambda}\left(u-m_{2}\right) \cdot F_{A}(u) \cdot H_{A}(u) .
$$

The convolution for the OLCT of two signals $f(t)$ and $h(t)$ associated with the Hermite functions $\phi_{n}^{\lambda}\left(\frac{u}{\sqrt{3}}-m_{2}\right)$ scaled by the chirp $e^{\frac{j m_{1} u}{\sqrt{3}}}$, is defined as

$$
(f \stackrel{3}{\otimes} h)(t):=\frac{\sqrt{3} K_{A}^{2} e^{j\left(m_{3}-\frac{d u_{0}^{2}}{2 b}\right)}\left(\mathcal{E}_{A}(t)\right)^{-1}}{\mu_{n}^{\lambda} \cdot \sqrt{\lambda}} \int_{\mathbb{R}^{2}} \bar{f}(\tau) \bar{h}(v) \cdot \mathcal{E}_{A}^{m_{1}}(\sqrt{3} t-\tau-v+\kappa) \phi_{n}^{\lambda}\left(\frac{\sqrt{3} t-\tau-v-m_{2}+\kappa}{\lambda}\right) d \tau d v,
$$

where $\kappa=(3-\sqrt{3})\left(b \omega_{0}-d u_{0}\right)$ (as long as the integral in (22) is well-defined). Moreover, if $f, h \in L^{1}(\mathbb{R})$ then $(f \stackrel{3}{\otimes} h)(t) \in L^{1}(\mathbb{R})$ since $\|f \stackrel{3}{\otimes} h\|_{1} \leqslant C_{3}\|f\|_{1} \cdot\|h\|_{1}$ for some $C_{3}>0$. 
Theorem 5 Let $f, h \in L^{1}(\mathbb{R}), F_{A}$ and $H_{A}$ denote the OLCT of the signals $f(t)$ and $h(t)$, with parameter A, respectively. The following factorization identity holds

$$
\mathbb{O}_{A}\{(f \stackrel{3}{\otimes} h)(t)\}(u)=e^{\frac{j m_{1} u}{\sqrt{3}}} \cdot \phi_{n}^{\lambda}\left(\frac{u}{\sqrt{3}}-m_{2}\right) \cdot F_{A}\left(\frac{u}{\sqrt{3}}\right) \cdot H_{A}\left(\frac{u}{\sqrt{3}}\right) .
$$

Moreover, if $e^{\frac{j m_{1} u}{\sqrt{3}}} \cdot \phi_{n}^{\lambda}\left(\frac{u}{\sqrt{3}}-m_{2}\right) \cdot F_{A}\left(\frac{u}{\sqrt{3}}\right) \cdot H_{A}\left(\frac{u}{\sqrt{3}}\right) \in \mathbb{O}_{A}\left(L^{1}(\mathbb{R})\right)$, then

$$
(f \stackrel{3}{\otimes} h)(t)=\mathbb{O}_{A^{-1}}\left\{e^{\frac{j m_{1} u}{\sqrt{3}}} \cdot \phi_{n}^{\lambda}\left(\frac{u}{\sqrt{3}}-m_{2}\right) \cdot F_{A}\left(\frac{u}{\sqrt{3}}\right) \cdot H_{A}\left(\frac{u}{\sqrt{3}}\right)\right\}(t) .
$$

Proof. $\quad$ Based on (9) and (16), we have

$$
\begin{aligned}
& e^{j m_{1} u} \cdot \phi_{n}^{\lambda}\left(u-m_{2}\right) \cdot F_{A}(u) \cdot H_{A}(u)=e^{j m_{1} u} \cdot \phi_{n}^{\lambda}\left(u-m_{2}\right) \cdot K_{A}^{2} e^{j\left(\frac{d}{b} u^{2}+\frac{2\left(b \omega_{0}-d u_{0}\right)}{b} u\right)} \int_{\mathbb{R}^{2}} \bar{f}(\tau) \bar{h}(v) e^{-\frac{j u \tau}{b}} e^{-\frac{j u v}{b}} d \tau d v \\
& =\frac{e^{j\left(m_{3}-\frac{d u_{0}^{2}}{2 b}\right)} K_{A}^{3}}{\mu_{n}^{\lambda} \cdot \sqrt{\lambda}} e^{3 j\left(\frac{d}{2 b} u^{2}+\frac{\left(b \omega_{0}-d u_{0}\right)}{b} u\right)} \int_{\mathbb{R}} e^{-\frac{j u t}{b}} \cdot \mathcal{E}_{A}^{m_{1}}(t) \cdot \phi_{n}^{\lambda}\left(\frac{t-m_{2}}{\lambda}\right) d t \int_{\mathbb{R}^{2}} \bar{f}(\tau) \bar{h}(v) e^{-\frac{j u \tau}{b}} e^{-\frac{j u v}{b}} d \tau d v
\end{aligned}
$$

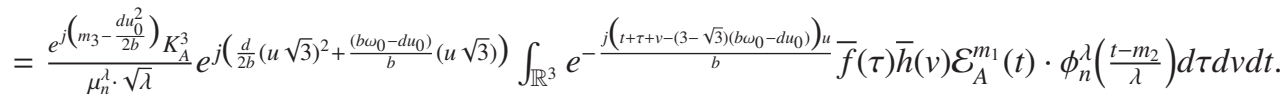

Performing the change of variables $\tau=\tau, v=v$ and $s=t+\tau+v-\kappa$, we achieve

$$
\begin{gathered}
e^{j m_{1} u} \phi_{n}^{\lambda}\left(u-m_{2}\right) \cdot F_{A}(u) \cdot H_{A}(u)=K_{A} e^{j\left(\frac{d}{2 b}(u \sqrt{3})^{2}+\frac{\left(b \omega_{0}-d u_{0}\right)}{b}(u \sqrt{3})\right)} \int_{\mathbb{R}} e^{-\frac{j u \sqrt{3}}{b}\left(\frac{s}{\sqrt{3}}\right)} \cdot \mathcal{E}_{A}\left(\frac{s}{\sqrt{3}}\right)\left\{\frac{K_{A}^{2} e^{j\left(m_{3}-\frac{d u_{0}^{2}}{2 b}\right)\left(\mathcal{E}_{A}\left(\frac{s}{\sqrt{3}}\right)\right)^{-1}}}{\mu_{n}^{\lambda} \cdot \sqrt{\lambda}} \times\right. \\
\left.\int_{\mathbb{R}^{2}} \bar{f}(\tau) \bar{h}(v) \cdot \mathcal{E}_{A}^{m_{1}}(s-\tau-v+\kappa) \cdot \phi_{n}^{\lambda}\left(\frac{s-\tau-v-m_{2}+\kappa}{\lambda}\right) d \tau d v\right\} d s \\
=\int_{\mathbb{R}} \mathcal{K}_{A}\left(u \sqrt{3}, \frac{s}{\sqrt{3}}\right)\left\{\frac{K_{A}^{2} e^{j\left(m_{3}-\frac{d u_{0}^{2}}{2 b}\right)}\left(\mathcal{E}_{A}\left(\frac{s}{\sqrt{3}}\right)\right)^{-1}}{\mu_{n}^{\lambda} \cdot \sqrt{\lambda}} \int_{\mathbb{R}^{2}} \bar{f}(\tau) \bar{h}(v) \cdot \mathcal{E}_{A}^{m_{1}}(s-\tau-v+\kappa) \phi_{n}^{\lambda}\left(\frac{s-\tau-v-m_{2}+\kappa}{\lambda}\right) d \tau d v\right\} d s \\
=\int_{\mathbb{R}} \mathcal{K}_{A}\left(u \sqrt{3}, \frac{s}{\sqrt{3}}\right) \cdot(f \stackrel{3}{\otimes} h)\left(\frac{s}{\sqrt{3}}\right) \cdot d\left(\frac{s}{\sqrt{3}}\right)=\mathbb{O}_{A}\{(f \stackrel{3}{\otimes} h)\}(u \sqrt{3}),
\end{gathered}
$$

which proves the theorem.

Corollary 1 Let $f, h \in L^{1}(\mathbb{R}), k \in\{1,2\}, F_{A_{1}}$ and $H_{A_{1}}$ denote the LCT of the signals $f(t)$ and $h(t)$ with parameters $A_{1}$, respectively. The convolution of two signals $f(t), h(t)$ for the LCT is defined as follows

$$
(f \stackrel{k}{\otimes} h)(t)=\frac{K_{A_{1}}^{2}\left(\mathcal{E}_{A_{1}}(t)\right)^{-1}}{\mu_{n}^{\lambda} \cdot \sqrt{\lambda}} \int_{\mathbb{R}^{2}} \bar{f}(\tau) \bar{h}(v) \cdot \overline{\phi_{n}^{\lambda}}\left(\frac{t-\tau-v}{\lambda}\right) d \tau d v .
$$

and we have

$$
\mathbb{O}_{A_{1}}\{(f \stackrel{k}{\otimes} h)(t)\}(u)=\phi_{n}^{\lambda}(u) \cdot e^{-j \frac{d}{2 b} u^{2}} \cdot F_{A_{1}}(u) \cdot H_{A_{1}}(u) .
$$

Corollary 2 Let $f, h \in L^{1}(\mathbb{R}), F_{A_{1}}$ and $H_{A_{1}}$ are the LCT of the signals $f(t)$ and $h(t)$ with parameters $A_{1}$. The convolution of two signals $f(t), h(t)$ for the LCT with the Hermite weights $\phi_{n}^{\lambda}\left(\frac{u}{\sqrt{3}}\right)$ is defined by

$$
(f \stackrel{3}{\otimes} h)(t)=\frac{\sqrt{3} K_{A_{1}}^{2}\left(\mathcal{E}_{A_{1}}(t)\right)^{-1}}{\mu_{n}^{\lambda} \cdot \sqrt{\lambda}} \int_{\mathbb{R}^{2}} \bar{f}(\tau) \bar{h}(v) \cdot \overline{\phi_{n}^{\lambda}}\left(\frac{\sqrt{3} t-\tau-v}{\lambda}\right) d \tau d v .
$$

and the following relation holds

$$
\mathbb{O}_{A_{1}}\left\{\left(f^{\otimes} \stackrel{3}{\otimes} h\right)(t)\right\}(u)=\phi_{n}^{\lambda}\left(\frac{u}{\sqrt{3}}\right) \cdot F_{A_{1}}\left(\frac{u}{\sqrt{3}}\right) \cdot H_{A_{1}}\left(\frac{u}{\sqrt{3}}\right) .
$$




\section{APPLICATIONS}

\section{Young's Convolution Inequalities}

Note that $1=\left|e^{j t}\right|=\left|\mathcal{E}_{A}(t)\right|=\left|\mathcal{E}_{A}^{m}(t)\right|=\left|\mu_{n}^{\lambda}\right|$, and $|f(t)|=|\bar{f}(t)|$. We derive the following theorem (see $\left.[1,3]\right)$.

Theorem 6 Suppose that $p, q, r, s \geqslant 1$, and $\frac{1}{p}+\frac{1}{q}=\frac{1}{r}+1, k \in\{1,2,3\}$. Then,

(i) $\|f \stackrel{k}{\otimes} h\|_{s} \leqslant C_{4}\left\|\phi_{n}\right\|_{s} \cdot\|f\|_{1} \cdot\|h\|_{1}$, for any $f, h \in L^{1}(\mathbb{R})$.

(ii) $\|f \stackrel{k}{\otimes} h\|_{r} \leqslant C_{5}\left\|\phi_{n}\right\|_{1} \cdot\|f\|_{p} \cdot\|h\|_{q}$, for any $f \in L^{p}(\mathbb{R}), h \in L^{q}(\mathbb{R})$, where $C_{1}, C_{2}$ are some positive constants.

Proof. We will present the proof for the case $k=3$. The cases $k \in\{1,2\}$ will be omitted because the proofs are analogous. Remind that $\phi_{n}(t)$ are rapidly decreasing functions. By applying the Minkowski's integral inequality and changing variable we obtain

$$
\begin{gathered}
{\left[\int_{\mathbb{R}}|(f \stackrel{k}{\otimes} h)(t)|^{s} d t\right]^{1 / s}=\left|\frac{\sqrt{3} K_{A}^{2}}{\sqrt{\lambda}}\right|\left[\int_{\mathbb{R}}\left|\int_{\mathbb{R}^{2}} \bar{f}(\tau) \bar{h}(v) \cdot \phi_{n}^{\lambda}\left(\frac{\sqrt{3} t-\tau-v-m_{2}+\kappa}{\lambda}\right) d \tau d v\right|^{s} d t\right]^{1 / s}} \\
\leqslant\left|\frac{\sqrt{3} K_{A}^{2}}{\sqrt{\lambda}}\right| \int_{\mathbb{R}^{2}}\left(\int_{\mathbb{R}}\left|\phi_{n}^{\lambda}\left(\frac{\sqrt{3} t-\tau-v-m_{2}+\kappa}{\lambda}\right)\right| s \cdot|\bar{f}(\tau)|^{s} \cdot|\bar{h}(v)|^{s} d t\right)^{1 / s} d \tau d v \\
=\left|\frac{\sqrt{3} K_{A}^{2}}{\sqrt{\lambda}}\right| \int_{\mathbb{R}^{2}}\left(\int_{\mathbb{R}}\left|\phi_{n}^{\lambda}\left(\frac{\sqrt{3} t-\tau-v-m_{2}+\kappa}{\lambda}\right)\right|^{s} d t\right)^{1 / s} \cdot|\bar{f}(\tau)| \cdot|\bar{h}(v)| d \tau d v \\
\leqslant C_{4}\left\|\phi_{n}\right\|_{s} \int_{\mathbb{R}^{2}}|f(\tau)| \cdot|h(v)| d \tau d v=C_{4}\left\|\phi_{n}\right\|_{s} \cdot\|f\|_{1} \cdot\|h\|_{1},
\end{gathered}
$$

where $C_{4}$ is a positive constant. Thus, we obtain $(i)$.

Now, we turn to the proof of (ii). Due to the formula (5) the convolution (22) can be also expressed as

$$
(f \stackrel{3}{\otimes} h)(t)=3 K_{A}\left(\mathcal{E}_{A}(t)\right)^{-1} \cdot\left(\bar{f}(\sqrt{3} t) * \bar{h}(\sqrt{3} t) * \mathcal{G}_{\kappa}(\sqrt{3} t)\right),
$$

where $\mathcal{G}_{\kappa}(t):=\frac{\sqrt{3} K_{A} e^{\left(m_{3}-\frac{d u_{0}^{2}}{2 b}\right)}}{\mu_{n}^{\lambda} \cdot \sqrt{\lambda}} \cdot \mathcal{E}_{A}^{m_{1}}(t+\kappa) \cdot \phi_{n}^{\lambda}\left(\frac{t-m_{2}+\kappa}{\lambda}\right)$. Remind that $f \in L^{p}(\mathbb{R}), h \in L^{q}(\mathbb{R})$. By performing a change of variable, we realize that $\bar{f}(\sqrt{3} t) \in L^{p}(\mathbb{R}), \bar{h}(\sqrt{3} t) \in L^{q}(\mathbb{R})$. Applying the Young's inequality (6) for the case $\frac{1}{p}+\frac{1}{q}=\frac{1}{r}+1$, we have $\bar{f}(\sqrt{3} t) * \bar{h}(\sqrt{3} t) \in L^{r}(\mathbb{R})$. Since the Hermite functions $\phi_{n}(t)$ are rapidly decreasing functions then, applying the Young's inequality (6) for the case $\frac{1}{r}+\frac{1}{1}=\frac{1}{r}+1$, we get $(\bar{f}(\sqrt{3} t) * \bar{h}(\sqrt{3} t)) * \mathcal{G}_{\kappa}(\sqrt{3} t) \in L^{r}(\mathbb{R})$. Moreover, we also achieve

$$
\|f \stackrel{k}{\otimes} h\|_{r} \leqslant C_{5}\left\|\phi_{n}\right\|_{1} \cdot\|f\|_{p} \cdot\|h\|_{q},
$$

where $C_{5}$ is a positive constant. The proof is completed.

\section{The Multiplicative Filter in the OLCT Domain}

In this subsection, we will discuss an application of the new convolution to the design of multiplicative filters in the OLCT domain (see [7]). We only consider the convolution (19) when $n=0$. The Hermite function $\phi_{0}^{\lambda}(t)$ and the value $\mu_{0}$ are given by

$$
\phi_{0}^{\lambda}(t)=\frac{1}{\sqrt{\beta \sqrt{\pi}}} e^{-\frac{(1+j \alpha)}{2 \beta^{2}} t^{2}}, \quad \mu_{0}=e^{\frac{-j \theta}{2}},
$$

where $\alpha, \beta, \theta$ can be taken from (8). We shall denote by $r_{\text {in }}(t)$ and $r_{\text {out }}(t)$ the input signal and output signal, respectively. From (20), the output signal can be expressed as

$$
r_{\text {out }}(t)=\mathbb{O}_{A^{-1}}\left\{\mathbb{O}_{A}\left\{r_{\text {in }}(t)\right\}(u) \cdot e^{j m_{1} u} e^{-j\left(\frac{d}{b} u^{2}+2 \frac{\left(b \omega_{0}-d u_{0}\right)}{b} u\right)} \cdot \phi_{0}^{\lambda}\left(u-m_{2}\right) \cdot H_{A}(u)\right\}(t) .
$$




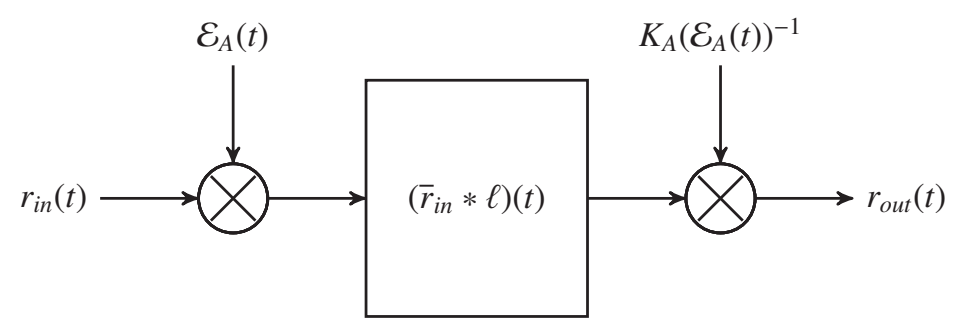

FIGURE 1. Method of achieving multiplicative filter in time domain.

Let us now denote

$$
\widetilde{H}_{A}(u)=e^{j m_{1} u} e^{-j\left(\frac{d}{b} u^{2}+2 \frac{\left(b \omega_{0}-d u_{0}\right)}{b} u\right)} \cdot \phi_{0}^{\lambda}\left(u-m_{2}\right) \cdot H_{A}(u) .
$$

Then, it follows $H_{A}(u)=e^{-j m_{1} u} e^{j\left(\frac{d}{b} u^{2}+2 \frac{\left(b \omega_{0}-d u_{0}\right)}{b} u\right)} \cdot\left(\phi_{0}^{\lambda}\left(u-m_{2}\right)\right)^{-1} \cdot \widetilde{H}_{A}(u)$.

Based on different transforms $\widetilde{H}(u)$, there are many ways to design a multiplicative filter. For instance, we can choose the function $h(t)$ such that $\widetilde{H}_{A}(u)$ is constant over $[-\Omega, \Omega]$, and zero or with rapid decay outside that region. Let $T$ be a constant and

$$
\widetilde{H}_{A}(u)= \begin{cases}T, & u \in[-\Omega, \Omega] \\ 0, & u \notin[-\Omega, \Omega]\end{cases}
$$

Thus, we obtain

$$
r_{\text {out }}(t)=T \cdot \mathbb{O}_{A^{-1}}\left\{\mathbb{O}_{A}\left\{r_{\text {in }}(t)\right\}(u)\right\}(t)
$$

From (3), the output signal $r_{\text {out }}(t)$ can be rewritten as

$$
r_{\text {out }}(t)=K_{A}\left(\mathcal{E}_{A}(t)\right)^{-1}\left(\bar{r}_{\text {in }} * \ell\right)(t)
$$

where the convolution function $\ell(t)$ is given by

$$
\ell(t)=\frac{K_{A} e^{j\left(m_{3}-\frac{d u_{0}^{2}}{2 b}\right)}}{\mu_{0}^{\lambda} \cdot \sqrt{\lambda}} \int_{\mathbb{R}} \bar{h}(v) \cdot \mathcal{E}_{A}^{m_{1}}(t-v) \cdot \phi_{0}^{\lambda}\left(\frac{t-v-m_{2}}{\lambda}\right) d v .
$$

This shows that we can achieve the multiplicative filter through the classic Fourier convolution of $r_{\text {int }}(t)$ and $\ell(t)$ in the time domain. A realization of the method is displayed in Figure 1 (see also [7]).

Using the expression (2), we obtain

$$
\begin{aligned}
h(t) & =C K_{A^{-1}} \int_{\mathbb{R}} H_{A}(u) e^{-j\left(\frac{d}{2 b} u^{2}-\frac{1}{b} t u+\frac{a}{2 b} t^{2}+\frac{\left(b \omega_{0}-d u_{0}\right)}{b} u+\frac{u_{0}}{b} t\right)} d u \\
& =C K_{A^{-1}} \int_{\mathbb{R}} e^{-j m_{1} u} e^{j\left(\frac{d}{b} u^{2}+2 \frac{\left(b \omega_{0}-d u_{0}\right)}{b} u\right)} \cdot\left(\phi_{0}^{\lambda}\left(u-m_{2}\right)\right)^{-1} \cdot \widetilde{H}_{A}(u) e^{-j\left(\frac{d}{2 b} u^{2}-\frac{1}{b} t u+\frac{a}{2 b} t^{2}+\frac{\left(b \omega_{0}-d u_{0}\right)}{b} u+\frac{u_{0}}{b} t\right)} d u \\
& =C K_{A^{-1}}\left(\mathcal{E}_{A}(t)\right)^{-1} \int_{\mathbb{R}} e^{-j m_{1} u} e^{j\left(\frac{d}{2 b} u^{2}+\frac{\left(b \omega_{0}-d u_{0}\right)}{b} u\right)} \cdot\left(\phi_{0}^{\lambda}\left(u-m_{2}\right)\right)^{-1} \cdot \widetilde{H}_{A}(u) e^{\frac{j u t}{b}} d u .
\end{aligned}
$$

Then,

$$
\bar{h}(t)=C K_{A^{-1}} \int_{\mathbb{R}} e^{-j m_{1} u} e^{j\left(\frac{d}{2 b} u^{2}+\frac{\left(b \omega_{0}-d u_{0}\right)}{b} u\right)} \cdot\left(\phi_{0}^{\lambda}\left(u-m_{2}\right)\right)^{-1} \cdot \widetilde{H}_{A}(u) e^{\frac{j u t}{b}} d u .
$$



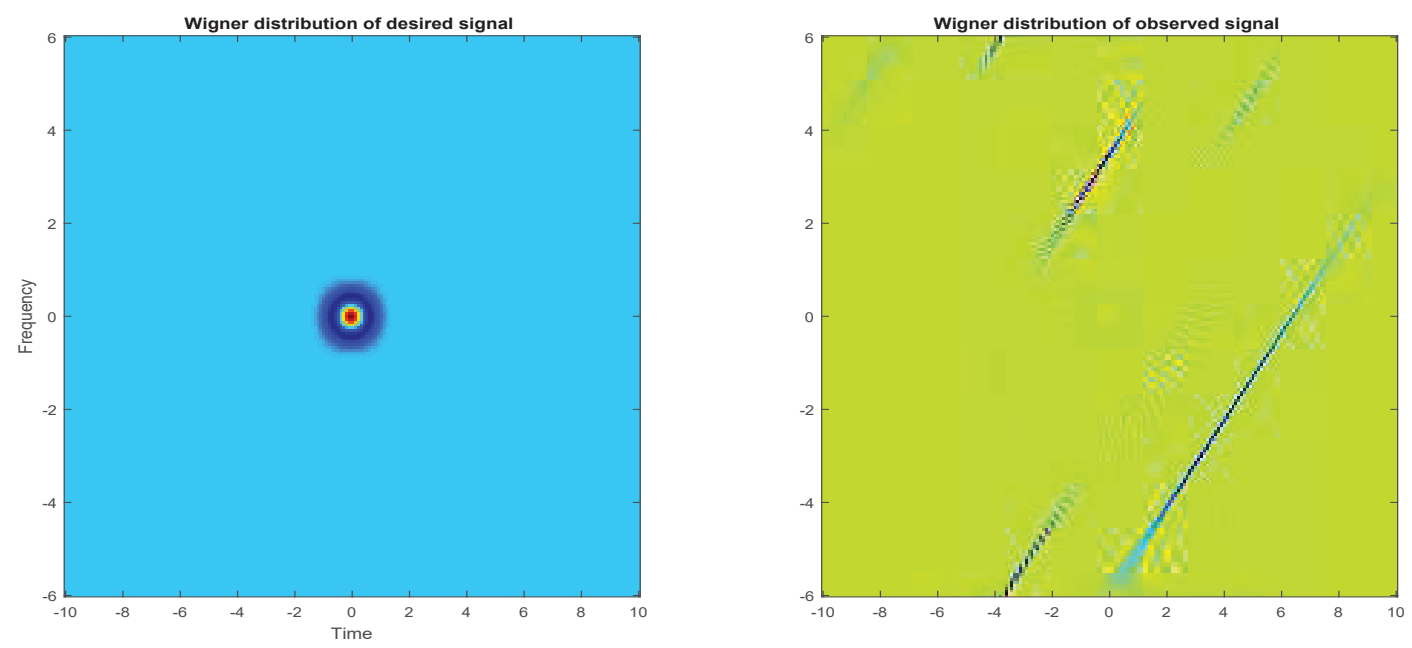

FIGURE 2. Wigner distributions of desired and observed signals.

Substituting (34) into (33), gives rise to

$$
\begin{aligned}
& \ell(t)=\frac{K_{A} e^{j\left(m_{3}-\frac{d u_{0}^{2}}{2 b}\right)}}{\mu_{0}^{\lambda} \cdot \sqrt{\lambda}} \int_{\mathbb{R}} \bar{h}(v) \cdot \mathcal{E}_{A}^{m_{1}}(t-v) \cdot \phi_{0}^{\lambda}\left(\frac{t-v-m_{2}}{\lambda}\right) d v \\
& =\frac{K_{A} e^{j\left(m_{3}-\frac{d u_{0}^{2}}{2 b}\right)}}{\mu_{0}^{\lambda} \cdot \sqrt{\lambda}} \int_{\mathbb{R}}\left(C K_{A^{-1}} \int_{\mathbb{R}} e^{-j m_{1} u} e^{j\left(\frac{d}{2 b} u^{2}+\frac{\left(b \omega_{0}-d u_{0}\right)}{b} u\right)} \cdot\left(\phi_{0}^{\lambda}\left(u-m_{2}\right)\right)^{-1} \widetilde{H}_{A}(u) e^{\frac{j u v}{b}} d u\right) \mathcal{E}_{A}^{m_{1}}(t-v) \cdot \phi_{0}^{\lambda}\left(\frac{t-v-m_{2}}{\lambda}\right) d v \\
& =\frac{C K_{A^{-1}} K_{A} e^{j\left(m_{3}-\frac{d u_{0}^{2}}{2 b}\right)}}{\mu_{0}^{\lambda} \cdot \sqrt{\lambda}} \int_{\mathbb{R}} \int_{\mathbb{R}} e^{-j m_{1} u} e^{j\left(\frac{d}{2 b} u^{2}+\frac{\left(b \omega_{0}-d u_{0}\right)}{b} u\right)} \cdot\left(\phi_{0}^{\lambda}\left(u-m_{2}\right)\right)^{-1} \cdot \widetilde{H}_{A}(u) e^{\frac{i u v}{b}} e^{j m_{1}(t-v)} \mathcal{E}_{A}(t-v) \cdot \phi_{0}^{\lambda}\left(\frac{t-v-m_{2}}{\lambda}\right) d v d u \\
& =\frac{C K_{A^{-1}} e^{j\left(m_{3}-\frac{d u_{0}^{2}}{2 b}\right)}}{\mu_{0}^{\lambda} \cdot \sqrt{\lambda}} \int_{\mathbb{R}}\left(e^{-j m_{1} u}\left(\phi_{0}^{\lambda}\left(u-m_{2}\right)\right)^{-1} \cdot \widetilde{H}_{A}(u)\right)\left(K_{A} e^{j\left(\frac{d}{2 b} u^{2}+\frac{\left(b \omega_{0}-d u_{0}\right)}{b} u\right)} \int_{\mathbb{R}} e^{\frac{j u v}{b}} e^{j m_{1}(t-v)} \mathcal{E}_{A}(t-v) \cdot \phi_{0}^{\lambda}\left(\frac{t-v-m_{2}}{\lambda}\right) d v\right) d u .
\end{aligned}
$$

By taking $s=t-v$, it shows that

$$
\begin{aligned}
K_{A} e^{j\left(\frac{d}{2 b} L^{2}+\frac{\left(b \omega_{0}-d u_{0}\right)}{b} u\right)} \int_{\mathbb{R}} e^{\frac{j w v}{b}} e^{j m_{1}(t-v)} \mathcal{E}_{A}(t-v) \phi_{0}^{\lambda}\left(\frac{t-v-m_{2}}{\lambda}\right) d v & =e^{\frac{j u t}{b}} K_{A} e^{j\left(\frac{d}{2 b} u^{2}+\frac{\left(b \omega_{0}-d u_{0}\right)}{b} u\right)} \int_{\mathbb{R}} e^{-\frac{j u s}{b}} \mathcal{E}_{A}(s)\left(e^{j m_{1} s} \phi_{0}^{\lambda}\left(\frac{s-m_{2}}{\lambda}\right)\right) d s \\
& =e^{\frac{j u t}{b}} \mathbb{O}_{A}\left\{e^{j m_{1} s} \phi_{0}^{\lambda}\left(\frac{s-m_{2}}{\lambda}\right)\right\}(u) .
\end{aligned}
$$

Manipulating (15), we have

$$
K_{A} e^{j\left(\frac{d}{2 b} u^{2}+\frac{\left(b \omega_{0}-d u_{0}\right)}{b} u\right)} \int_{\mathbb{R}} e^{\frac{j u v}{b}} e^{j m_{1}(t-v)} \mathcal{E}_{A}(t-v) \cdot \phi_{0}^{\lambda}\left(\frac{t-v-m_{2}}{\lambda}\right) d v=\mu_{0}^{\lambda} \cdot \sqrt{\lambda} e^{-j\left(m_{3}-\frac{d u_{0}^{2}}{2 b}\right)} e^{\frac{j u t}{b}} e^{j m_{1} u} \cdot \phi_{0}^{\lambda}\left(u-m_{2}\right) .
$$

Therefore, $\ell(t)=C K_{A^{-1}} \int_{\mathbb{R}} \widetilde{H}_{A}(u) e^{\frac{j u t}{b}} d u$. Based on the relation (31), we derive

$$
\ell(t)=C K_{A^{-1}} \int_{-\Omega}^{\Omega} T e^{\frac{j u t}{b}} d u=2 b C K_{A^{-1}} T \cdot \frac{\sin \left(\frac{t \Omega}{b}\right)}{t} .
$$

Hence $\ell(t)=\frac{T}{\pi K_{A}} \cdot \frac{\sin \left(\frac{t \Omega}{b}\right)}{t}$.

In the following example, we shall use the proposed multiplicative filter to restore an observed signal $r_{i n}(t)=$ $X(t)+N(t)$, where $X(t), N(t)$ denote the desired signal and the additive noise, respectively. 

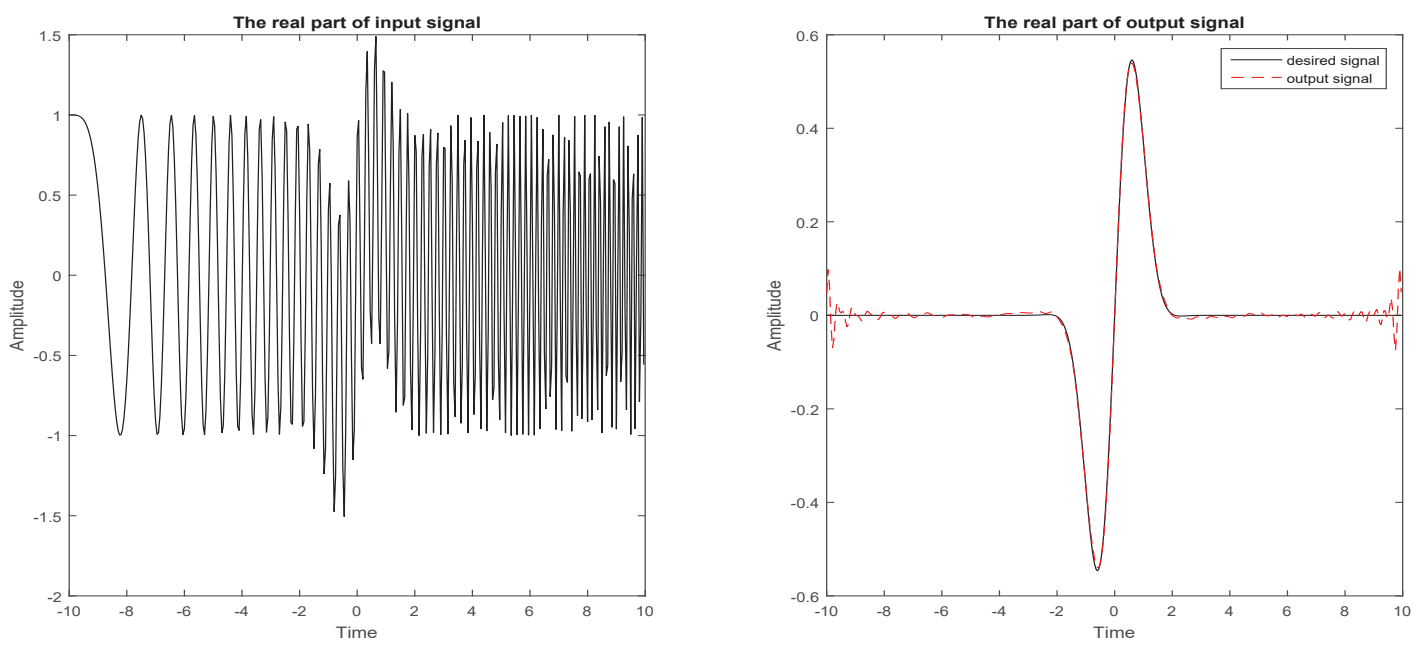

FIGURE 3. Result of multiplicative filter achieved by using convolution (19).

Example 2 We use $r_{i n}(t)=e^{-t^{2}} \cdot \sin (1.5 t)+e^{i(t+10)^{2}}, X(t)=e^{-t^{2}} \cdot \sin (1.5 t)$, and $N(t)=e^{i(t+10)^{2}}$. For convenience, let $u_{0}=\omega_{0}=0$. The Wigner distributions of $X(t)$ and $r_{i n}(t)$ are shown in Figure 2. Thus, we can choose (see [5]) $a=-\frac{2}{3}, b=\frac{1}{3}, \Omega=2$. The transfer function reads

$$
\widetilde{H}_{A}(u)= \begin{cases}1, & u \in[-2,2] \\ 0, & u \notin[-2,2],\end{cases}
$$

and $\ell(t)=\sqrt{\frac{2 i}{3 \pi}} \cdot \frac{\sin 6 t}{t}$. The output signal can be expressed as $r_{\text {out }}(t)=\sqrt{\frac{3}{2 \pi i}} e^{i t^{2}} \cdot\left(\bar{r}_{i n} * \ell\right)(t)$. The consequent result of the multiplicative filter is displayed in Figure 3.

\section{ACKNOWLEDGMENTS}

This work was supported in part by FCT-Portuguese Foundation for Science and Technology through the Center for Research and Development in Mathematics and Applications (CIDMA) of Universidade de Aveiro, within project UID/MAT/04106/2013, and by the Viet Nam National Foundation for Science and Technology Development (NAFOSTED).

\section{REFERENCES}

[1] P. K. Anh, L. P. Castro, P. T. Thao and N. M. Tuan, Inequalities and consequences of new convolutions for the fractional Fourier transform with Hermite weights, American Institute of Physics, AIP Proceedings 1798(1), (2017).

[2] W. Beckner, Inequalities in Fourier analysis, Annals of Math 102(1), 159-182 (1975).

[3] L. P. Castro, L. T. Minh and N. M. Tuan, New convolutions for quadratic-phase Fourier integral operators and their applications, Mediterranean Journal of Mathematics 15:13, 17pp. (2018).

[4] A. Koc, H. M. Ozaktas, C. Candan and M. A. Kutay, Digital computation of linear canonical transforms, IEEE Trans. Signal Process 56(6) 2383-2394 (2008).

[5] S. C Pei and J. J. Ding, Relations between fractional operations and time-frequency distributions, and their applications, IEEE Trans. Signal Processing 49(8), 1638-1655 (2001).

[6] S. C. Pei and J. J. Ding, Eigenfunctions of linear canonical transform, IEEE Trans. Signal Process 50(1) 11-26 (2002).

[7] Q. Xiang and K. Qin, Convolution, correlation, and sampling theorems for the offset linear canonical transform, Signal, Image and Video Processing 8(3), 433-442 (2014). 